\title{
Design of Missle Borne TV Guidance Image Processing Semi-physical Simulation Platform
}

\author{
Mengda Liu ${ }^{1, a}$, Xiong Chen ${ }^{2, b}$ and Kun $\mathrm{He}^{2, \mathrm{c}}$ \\ ${ }^{1}$ Nanjing University of Science and Technology, Nanjing 210094, China; \\ ${ }^{2}$ Nanjing University of Science and Technology, Nanjing 210094, China. \\ aa7692661@qq.com, bchenxiongsc@163.com, 654673991@qq.com
}

Keywords: Image processing, Target detection and tracking, Camshift, Difference method, DSP.

\begin{abstract}
Real-time detection and tracking of moving target is an important part in modern warfare, its authenticity and real-time quality determine the success or failure of the war. This article has designed a DSP based moving target detection and tracking semi-physical simulation system in the application background of conventional missile guidance. A modified difference method is proposed to detect and track moving targets in combination with the Camshift algorithm. It makes up for the lack of a single algorithm. And designs image processing platform with DM642 as the core and the camera automatic tracking control pan-tilt, Finally, the simulation of the system is carried out, Experimental results show that the system has good robustness and real-time performance.
\end{abstract}

\section{Introduction}

Computer vision is a very important part of modern high tech local war, and it plays a great role in many fields of military defense. With the advantages of high precision, high power and strong mobility, the missile using the precision guidance technology is gradually being favored by the countries all over the world. Under this background, the TV guidance [1] is a kind of precise guidance technology, which has important practical significance for the research of the core target detection and tracking system. Therefore, this paper designs a DM642 [2] based image processing platform, including video capture module, memory module, image processing module, communication module and so on. And through the tracking control platform achieve real-time tracking of moving target.

In this paper, the designed platform is DM642 to process the collected image, combined with the improved differential algorithm and Camshift [3, 4] algorithm to obtain the edge contour and motion information of the moving object. And pass the information obtained to control platform $[5,6]$ to drive motor to control camera to real time tracking of moving target. So this system should include two parts: image processing platform and tracking control platform.

\section{Principle of moving object detection and tracking algorithm}

Moving object detection and tracking based on image sequences is the core of TV imaging guidance technology. In recent decades, image processing technology has developed rapidly which has formed a variety of categories of moving target detection and tracking algorithm [7]. It is important to choose what kind of technology to deal with the image, which is related to whether it can accurately detect or accurately target the strategic target.

Moving object detection. The most commonly used moving object detection method is the frame difference method [8], and frame difference method is to detect the moving object by the difference between the two adjacent frames in the image sequence. The formula is as follows.

$$
\begin{aligned}
& D_{k}(x, y)=\left|f_{k}(x, y)-f_{k-1}(x, y)\right| \\
& T_{k}(x, y)= \begin{cases}1 & D_{k}(x, y) \geq T \\
0 & D_{k}(x, y)<T\end{cases}
\end{aligned}
$$


Among them, $f_{k}(x, y)$ and $f_{k-1}(x, y)$ represent the pixel gray value of the first $\mathrm{k}$ frame and the second k-1 frame image at (x, y) points. The difference of adjacent frames is expressed by $D_{k}(x, y)$. Binary images are represented by $T_{k}(x, y) . T$ is the set threshold.

Frame difference method is characterized by simple calculation, fast speed, and is not sensitive to changes in ambient light. But this method is difficult to achieve in the complex background.

In this paper, we use the combination of the improved symmetrical difference method and the background subtraction method. Using a continuous three frame image $\left(f_{\mathrm{k}-1}, f_{\mathrm{k}}, f_{\mathrm{k}+1}\right)$ and the background image $\left(b_{\mathrm{k}}\right)$, the obtained symmetrical difference image and background subtraction image directly multiplied to get the gray image. At the same time, the method of adaptive threshold segmentation is adopted to get the binary image, and finally the moving object is obtained. The moving object obtained by this method makes up for the lack of a single method, which has better real-time performance. The specific process as shown in Figure 1 below.

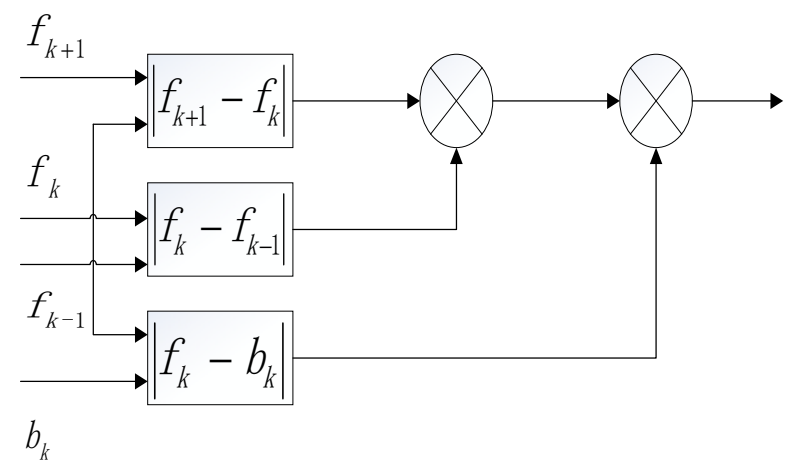

Fig. 1 Moving object detection model

Background image with light, time and other changes in the slow change, always use a constant background will inevitably affect the detection results. In order to reduce the impact of background on the results, this paper uses the following methods to update the background model in real time. Among them, the value of $\alpha$ is $0.4 \%$.

$$
B_{k+1}(x, y)= \begin{cases}B_{k}(x, y) & D_{k}(x, y)=1 \\ (1-\alpha) B_{k}(x, y)+\alpha f_{k}(x, y) & D_{k}(x, y)=0\end{cases}
$$

Moving object tracking. The position of the target in the image is obtained by moving object detection. From this, we can determine the initial search box size of the target tracking, and then we can calculate the motion vector of the target with the target tracking algorithm. The information is fed back to the control platform, so as to drive the camera to achieve the target tracking.

In view of the lack of necessary updates in the tracking process of the Meanshift algorithm tracking model, the tracking window size can't be changed according to the change of the target size. In this paper, we use the popular Camshift algorithm to track the target. The basic principle is to use the color information as tracking feature, color feature is projected to the next frame image, and the target area of the frame image is calculated by analyzing and matching according to the characteristic information. Then this frame image is used as the source graph, repeat the steps, continue to analyze the next frame image, so that it can realize the continuous tracking of the specific target. Camshift algorithm is using Meanshift iteration to find the optimal results of each image, when the target size is changed, it can effectively detect the moving target, make up for the shortcomings of Meanshift algorithm, the windows update methods are as follows.

$$
\begin{gathered}
M_{(0,1,2,3)}=\sum_{x} \sum_{y}\left(1, x^{2}, x y, y^{2}\right) I(x, y) \\
a=\frac{M_{1}}{M_{0}}-x_{c}^{2}, \quad b=2\left(\frac{M_{2}}{M_{0}}-x_{c} y_{c}\right), \quad c=\frac{M_{3}}{M_{0}}-y_{c}^{2}
\end{gathered}
$$




$$
\begin{aligned}
& d=\sqrt{\frac{(a+c)+\sqrt{b^{2}+(a-c)^{2}}}{2}} \\
& h=\sqrt{\frac{(a+c)-\sqrt{b^{2}+(a-c)^{2}}}{2}}
\end{aligned}
$$

Among them, $\mathrm{M}$ is the search window second moments, $\mathrm{d}$ and $\mathrm{h}$ respectively for the width and height of next frame search window.

In this paper, the traditional Camshift algorithm has been improved, through the combination of the difference method and the Camshift algorithm to solve the single algorithm need to manually initialize the tracking window size. Through the difference method to determine the moving area of the target, with its bounding rectangle as the initial tracking window to achieve real-time automatic tracking; By the motion estimation of the target, the position and size of the target search window are adjusted appropriately, which increases the effectiveness of the tracking.

\section{Hardware and software design of the system}

Target detection and tracking system can be divided into two parts, hardware platform and software algorithm, the normal operation of the software algorithm needs the support of the hardware platform, and the function of the system needs to mature software algorithm to achieve. In addition to the requirements of the hardware processing platform with high performance, the software algorithm will also affect the final results of the target detection and tracking system.

According to the function and algorithm used in this paper, the hardware design mainly includes two parts, image processing platform and tracking control platform. Includes CMOS camera, DM642, motion controller F2812, rotary platform.

The image processing platform mainly consists of video capture module, memory module, image processing module and communication module. First CMOS camera capture video images, the collected images are saved to the SDRAM; On one hand, DM642 will transfer state information of the target to PC for display, on the other hand, the image is processed by frame difference method to get the edge and outline of moving objects and determine the tracking window, using Camshift algorithm to track target and determine object position in the image and calculate the target motion information; Finally transfer motion information through the serial port to the tracking control platform.

According to the motion information, tracking control platform converted it into control signal of the motor to drive. Control camera tracking target. General structure of the system as shown in Figure 2 below.

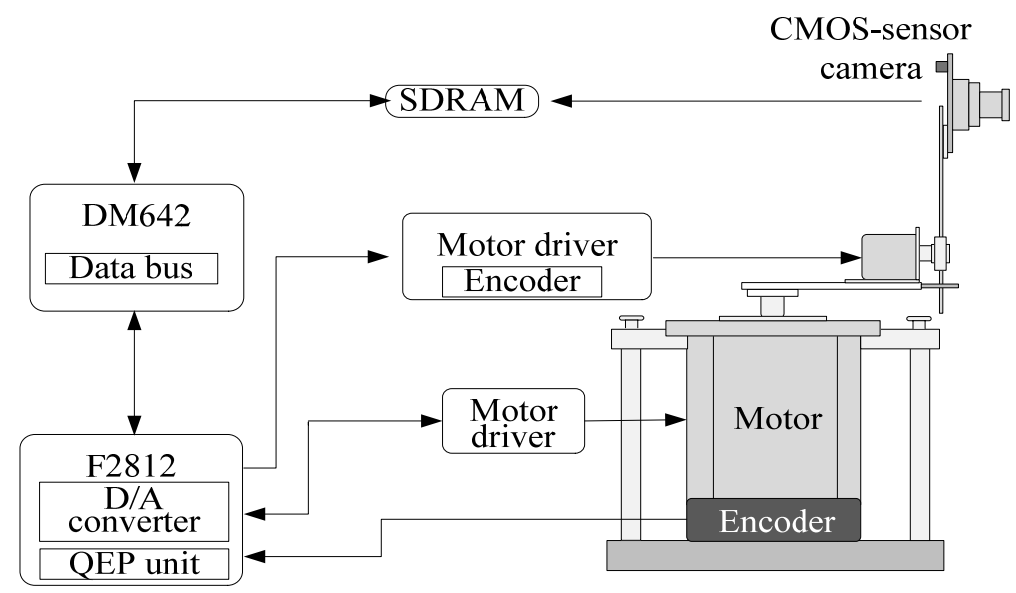

Fig. 2 General structure of the system

The software part is mainly divided into two parts, the development of the system software and the realization of the target detection and tracking algorithm. System software development including system initialization settings, as well as the design of each module and the main program; 
Implementation of the algorithm, including the preparation and transplantation of $\mathrm{C}$ language algorithm. Debug interface as shown in figure 3.

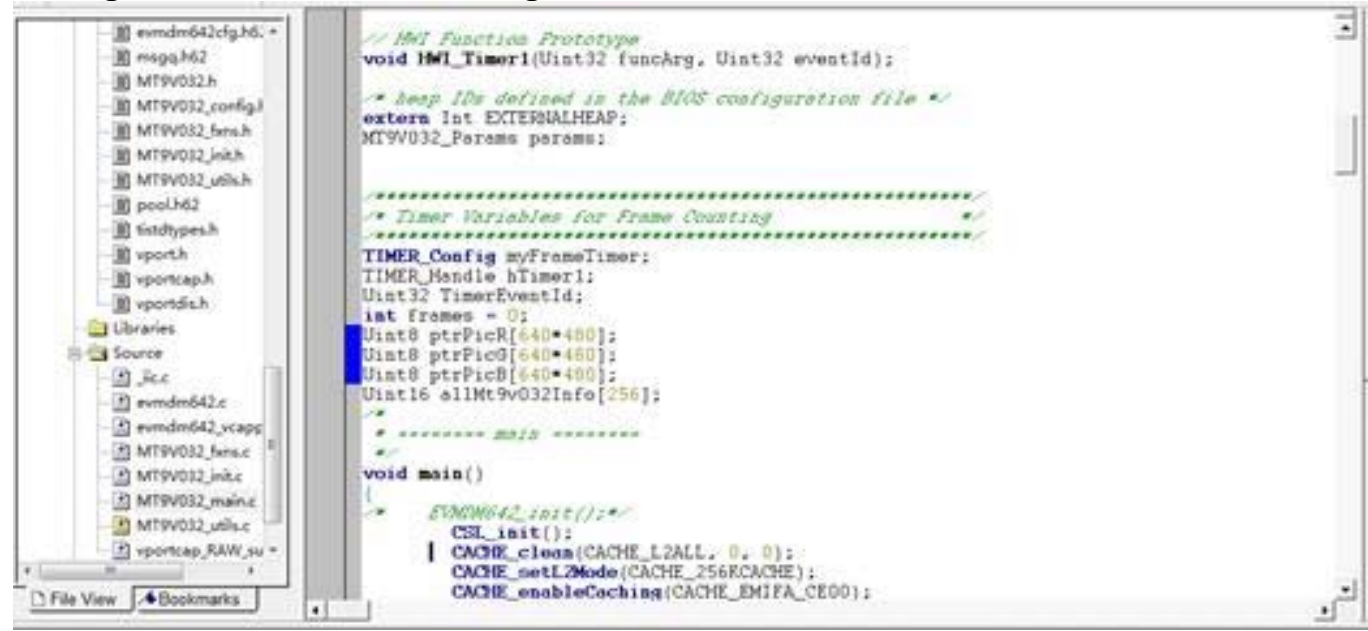

Fig. 3 Software debug interface

\section{Experimental data and analysis}

In this paper, the experimental simulation is carried out on the platform, the experimental object for a group of continuous video images, the experiment using the frame frequency of 30 frames / sec, the video image size of $640 \times 480$. The time to process a frame image is about $35 \mathrm{~ms}$. The experimental platform is shown in figure 4.

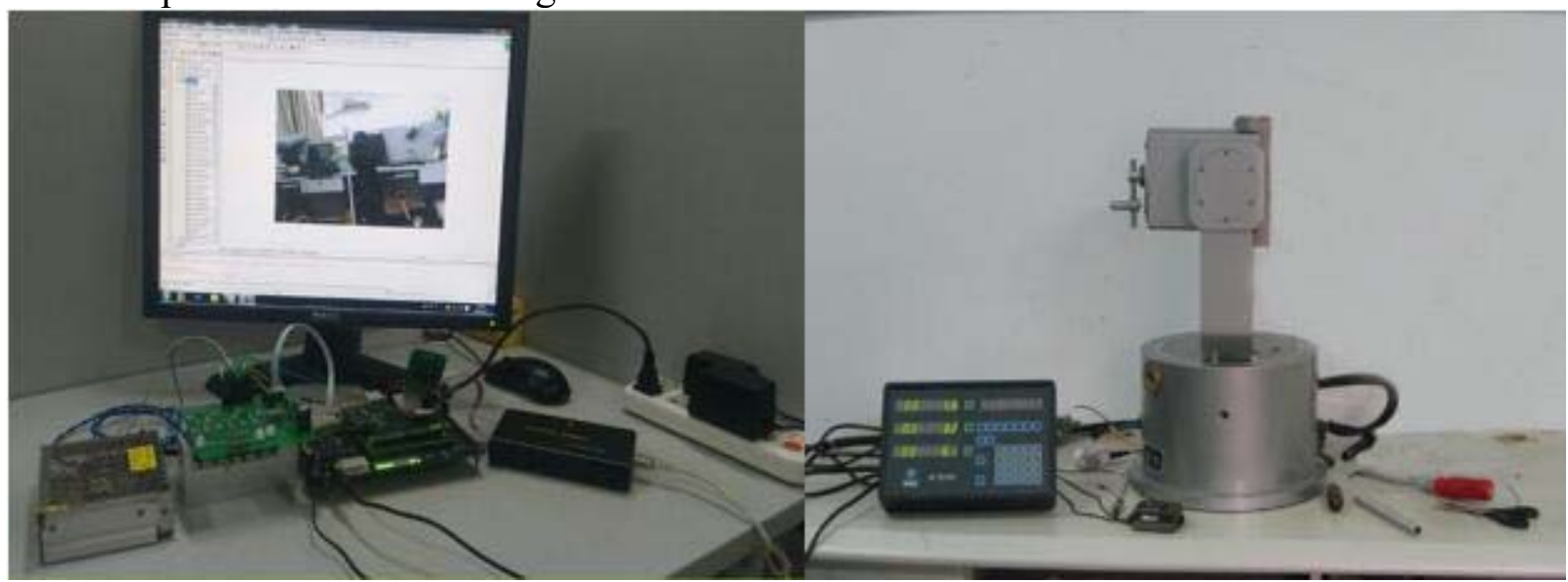

Fig. 4 DSP control system and tracking control platform

The combination of symmetrical difference method and background subtraction method. For the experiment of a video, a continuous three frame image is extracted, and the proposed method is used to detect the moving object. As shown in Figure 5 below. (1) (2) (3) are the continuous three frames of images, (4) for the background image, (5) for the proposed algorithm to detect the moving target (6) for the binary image of the moving target. It can be seen from the experimental results that the target detection method can be used to detect moving objects.

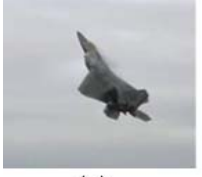

(1)

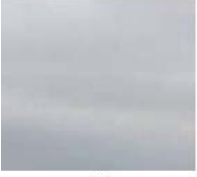

(4)

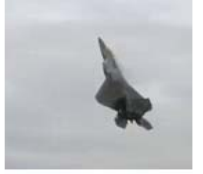

(2)

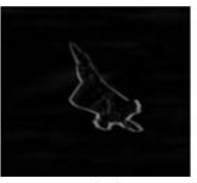

(5)
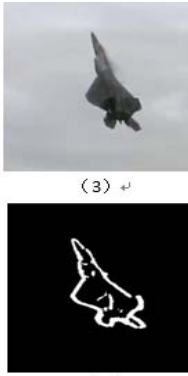

(6)

Fig. 5 Tracking detection experiment 
Experimental comparison between Camshift algorithm and Meanshift algorithm. In order to verify the effectiveness of the tracking algorithm, we use the Meanshift algorithm and the improved Camshift algorithm proposed in this paper to deal with the same video. The experimental results are shown in Figure 6 and Figure 7. It can be seen that the improved Camshift algorithm proposed in this paper is better than the Meanshift algorithm. Tracking window of Meanshift algorithm can't be changed with the target, and from the 194th frame, due to the impact of cloud and air flow, Meanshift algorithm has become very poor. The target location is not accurate. The algorithm of this paper has a very good tracking of the target. Tracking window can follow the tracking target size change and change the size of the tracking window in real time.

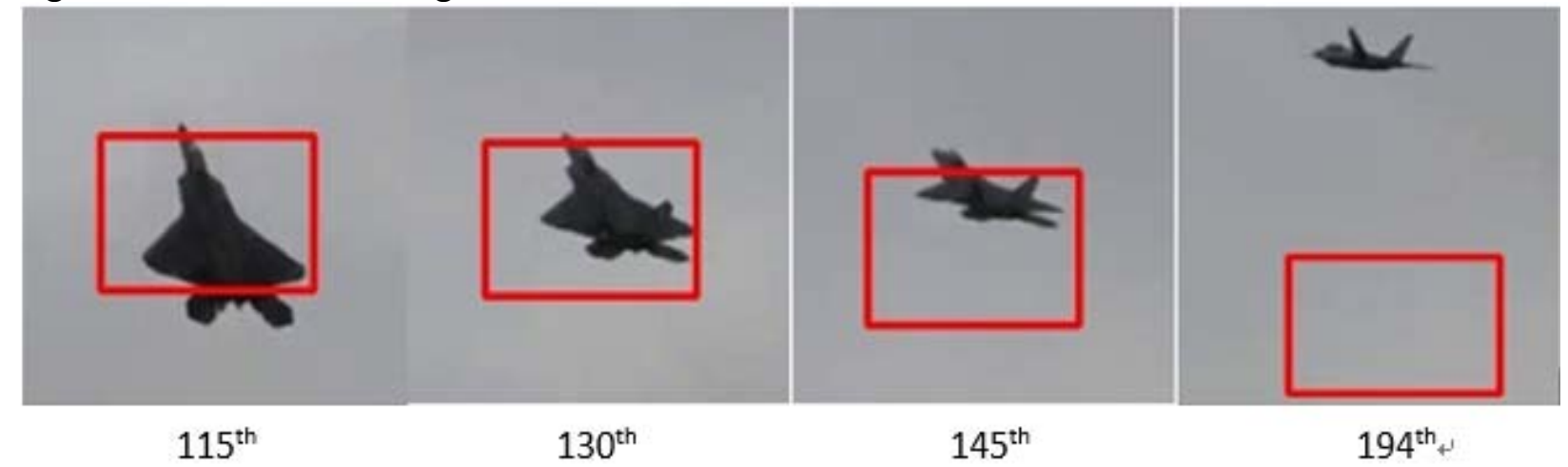

Fig. 6 Target tracking based on Meanshift algorithm

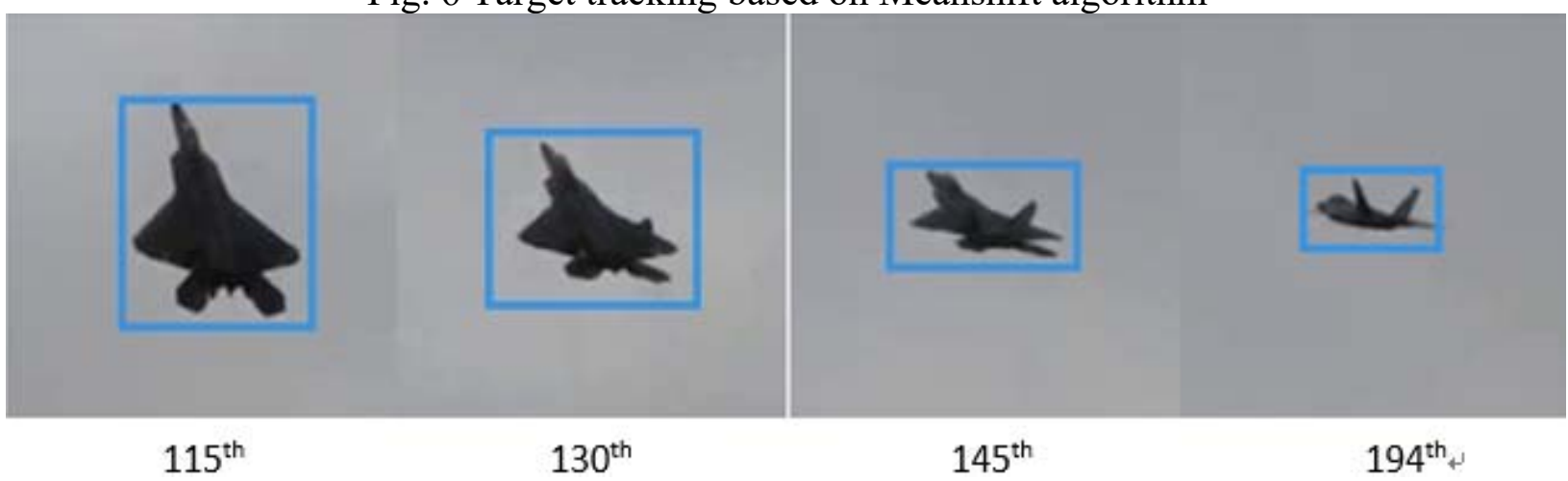

Fig. 7 Target tracking based on Camshift algorithm

Platform simulation experiment. Platform simulation results are shown in Figure 8, Table 1 and Figure 9. As can be seen from Figure 8, in the process of tracking, the unit time movement offset is larger, the tracking position is accurate and robust, and can be used in real-time video detection and tracking system; You can see from Table 1, the hardware design scheme of the measurement and tracking algorithm in most cases relative distance error in the range of 5\%, the maximum distance relative error is less than $10 \%$, so the measurement accuracy can meet the basic requirements of TV guidance. Figure 9 for the algorithm computing time in this paper, target tracking from about 100th frames, it can be seen that the algorithm used in this paper to deal with a frame of image needs time is only about $6 \mathrm{~ms}$, fully meet the requirements of real-time tracking.
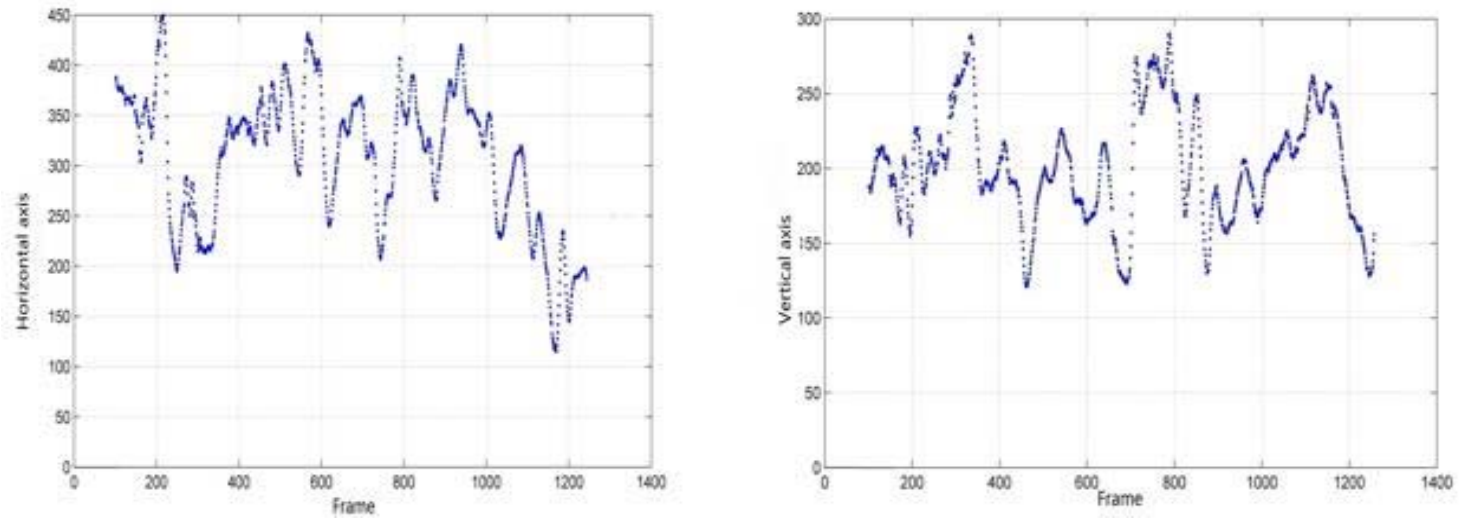

Fig. 8 Horizontal and vertical coordinate of target center 
Table 1 Comparison of target center error

\begin{tabular}{ccccccc}
\hline $\begin{array}{c}\text { Frame } \\
\text { number }\end{array}$ & \multicolumn{2}{c}{ Pixel coordinates } & \multicolumn{2}{c}{ Actual coordinates } & $\begin{array}{c}\text { measurement error } \\
\sqrt{\Delta x^{2}+\Delta y^{2}}\end{array}$ & $\begin{array}{c}\text { Relative } \\
\text { distance error }\end{array}$ \\
\hline 200 & 361 & 156 & 352 & 165 & 12.73 & $3.3 \%$ \\
400 & 322 & 203 & 313 & 204 & 9.06 & $2.4 \%$ \\
600 & 389 & 177 & 400 & 179 & 11.18 & $2.6 \%$ \\
800 & 338 & 242 & 327 & 257 & 18.60 & $4.5 \%$ \\
1000 & 325 & 170 & 333 & 166 & 8.94 & $2.4 \%$ \\
1200 & 148 & 168 & 162 & 172 & 14.56 & $6.2 \%$ \\
\hline
\end{tabular}

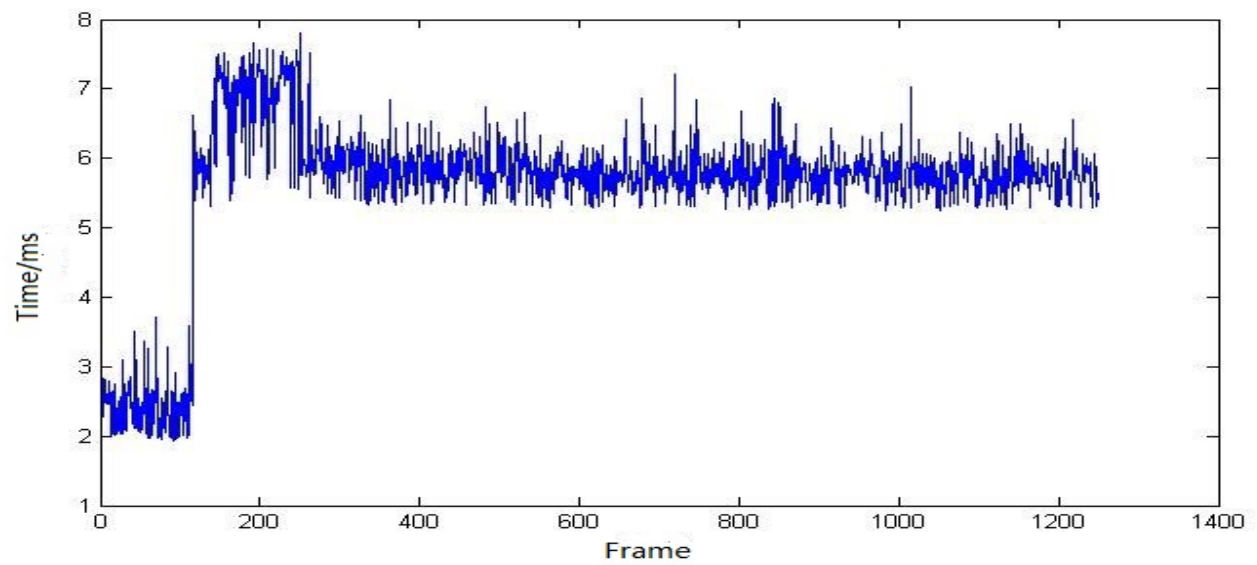

Fig. 9 Algorithm computing time

\section{Summary}

In this paper, we propose an improved method for the problem of difference method and Camshift algorithm, and a complete set of moving target detection and tracking platform is designed. The experiments show that the improved algorithm has a better tracking ability than the original algorithm. The system has strong robustness and good real-time performance. It can be used as a simulation platform for further research.

\section{References}

[1] Liu Xiaofeng, A DSP based real-time vision tracking system[J]. Optical technology, 2006,32(z1): 85-89.

[2] TMS320DM642 Digital Signal Processor Data Manual. Texas Instrument[M], 2005.

[3] Wu Dapeng, Chen Weiping, Yu Shenglin, Camshift Object Tracking Algorithm Based on Inter-frame Difference and Motion Prediction[J]. Opto electronic engineering, 2010, 37(1):55-60.

[4] Zhang Yang, Zhang Santong. Method of Multiple Moving Object Detection and Tracking Based on Self-adaptive Difference[J]. Modern electronic technology, 2010, 34(16):149-151.

[5] Zhang Jiancang, Yang Zhehui, Sun Qifu, Target Tracking System Design Based on Two Degrees of Freedom PTZ[J]. Computer measurement and control, 2013, 21(9):2579-2581.

[6] Tu Yiwei, Ming-Tzu Ho. Design and implementation of robust visual servoing control of an inverted pendulum with FPGA-based an image co-processor[J]. Mechatronics, 2010, 21(7):83-88.

[7] Hu Hongyu, Zhao Wei Qu, Li Zhihui, Wang Qingnian. Robust Detection and Tracking Algorithm of Multiple Objects in Complex Scenes[J].Applied Mathematics and Information Sciences, 2014, 8(5): 2485-2490.

[8] Zeng Wanmei. Research on fast target recognition and tracking technology based on image[D]. Nanjing: Nanjing University of Aeronautics \& Astronautics, 2009. 